\title{
Long non-coding RNA maternally expressed gene regulates cigarette smoke extract induced lung inflammation and human bronchial epithelial apoptosis via miR-149-3p
}

\author{
ZHENWU LEI ${ }^{1}$, HUI GUO ${ }^{2}$, SHENCHUN ZOU $^{2}$, JING JIANG $^{1}$, YUCHUAN KUI $^{3}$ and JIE SONG $^{3}$ \\ ${ }^{1}$ Department of Intervention, Qinghai University Affiliated Hospital, Xining, Qinghai 810000; \\ ${ }^{2}$ Department of Respiratory Medicine, Zaozhuang Mining Group Central Hospital, Zaozhuang, Shandong 277000; \\ ${ }^{3}$ Department of Thoracic Surgery, Dalian University Affiliated Xinhua Hospital, Dalian, Liaoning 116000, P.R. China
}

Received September 29, 2019; Accepted July 29, 2020

DOI: $10.3892 /$ etm.2020.9492

\begin{abstract}
Chronic obstructive pulmonary disease (COPD) has become a significant public health risk. Long non-coding RNAs (lncRNAs) have been identified as important factors involved in the proliferation, apoptosis and inflammatory cytokine expression of lung cells. Peripheral blood samples from 66 subjects (18 non-smokers, 24 smokers without COPD and 28 smokers with COPD) and HBE135-E6E7 cell treated with cigarette smoke extract (CSE) or not were used as the research object. The aim of the present study was to investigate the underlying mechanism of lncRNA maternally expressed gene 3 (MEG3) in COPD. Following transfection with microRNA (miR)-149-3p mimics, miR-negative control mimics, miR-149-3p inhibitor, miR-negative control inhibitor, small interfering (si)RNA targeting MEG3 (si-MEG3) and si-negative control (si-NC), levels of MEG3 and microRNA (miR)-149-3p were detected using reverse transcription-quantitative PCR, Proliferation and apoptosis were examined using the Cell Counting Kit- 8 and flow cytometry assays, respectively. Enzyme-linked immunosorbent assay (ELISA) was performed to detect the expression of interleukin-6 (IL-6) and tumor necrosis factor- $\alpha$ (TNF- $\alpha$ ). Protein levels of B-cell lymphoma-2 (Bcl-2), cleaved-caspase-3, cleaved-caspase-9, phosphorylated (p)-p65, total (t)-p65, p-lkB $\alpha$ and t-lkB $\alpha$ were measured by western blotting. Luciferase assay was conducted to examine the relationship between MEG3 and miR-149-3p. LncRNA MEG3 was highly expressed, whereas miR-149-3p expression was downregulated in smokers with COPD
\end{abstract}

Correspondence to: Dr Yuchuan Kui or Dr Jie Song, Department of Thoracic Surgery, Dalian University Affiliated Xinhua Hospital, 156 Wansui Street, Dalian, Liaoning 116000, P.R. China

E-mail:kuiyuchuandl@sina.com

E-mail: bentong41@yeah.net

Key words: long non-coding RNA maternally expressed gene 3, microRNA-149-3p, chronic obstructive pulmonary disease, apoptosis, inflammation peripheral blood samples, compared with non-smokers and smokers without COPD samples. Compared with untreated human bronchial epithelial (HBE) cells, MEG3 expression was increased in cigarette smoke extract (CSE)-treated HBE cells. Compared with CSE-treated HBE cells transfected with si-NC, MEG3 knockdown promoted cell proliferation and inhibited apoptosis in CSE-treated HBE cells transfected with si-MEG3, and it also decreased the levels of IL-6, TNF- $\alpha$, Bcl-2 and increased cleaved-caspase- 3 and cleaved-caspase- 9 in CSE-treated HBE cells transfected with si-MEG3. The luciferase assay demonstrated that miR-149-3p has target sites for MEG3. MEG3 was demonstrated to regulate the NF- $\kappa B$ signaling pathway by sponging miR-149-3p in CSE-treated HBE cells. In conclusion, these findings suggested that MEG3 promoted proliferation and inhibited apoptosis by regulating the NF- $\kappa B$ signal pathway via miR-149-3p in CSE-treated HBE cells. These results provide an insight for further verification and understanding of the molecular basis of COPD.

\section{Introduction}

Chronic obstructive pulmonary disease (COPD) has become a significant risk of public health, especially in adults aged $>70$ years $(1,2)$. In $2010, \sim 11.7 \%$ of people aged $\geq 30$ years were diagnosed with COPD worldwide (3). According to the data from the Institute for Health Metrics and Evaluation in 2017, the number of deaths globally due to COPD is $\sim 3$ million every year; in 2030, it is predicted that COPD will be the third most common disease of death worldwide (4). Subjects with COPD exhibit a number of symptoms, such as breathlessness and persistent productive coughing (5). Smoking is the leading risk factor for $\mathrm{COPD}$, although according to 14 countries from the international Burden of Obstructive Lung Disease study, $20 \%$ of non-smokers were diagnosed with COPD in 2008 (6). However, the standard treatment methods for COPD, such as cessation of smoking, may result in anxiety and depression in smokers (7). Thus, research into the underlying mechanism of how smoking influences COPD is essential for the development of treatments for the disease.

Long non-coding RNAs (lncRNAs) are $>200$ nucleotides long and are important regulators of various biological processes, 
including cell proliferation, apoptosis, and immunization (8-10). An increasing number of studies have demonstrated that lncRNAs are aberrantly expressed in COPD and are involved in cell inflammatory response and apoptosis (11-13). The expression of sphingosine-1-phosphate receptor 1 is promoted by the lncRNA long intergenic noncoding RNA antisense to S1PR1 to increase angiogenic capacity by regulating the sphingosine-1-phosphate signaling pathway in human umbilical vein endothelial cells (14). The lncRNA maternally expressed gene 3 (MEG3) has been identified as a tumor inhibitor, which suppresses the apoptosis and migration of cancer cells (15). Additionally, MEG3 is significantly increased in lung tissues of subjects with COPD compared with non-COPD lung tissues (16). In addition, a recent study has indicated that knockdown of MEG3 inhibits human bronchial epithelial (HBE) cell apoptosis and autophagy by regulating the expression of p53 (17). However, the exact role and underlying mechanism of lncRNA MEG3 in COPD requires further elucidation.

MicroRNAs (miRNAs/miRs) are non-coding RNA that serve vital roles in silencing or cleaving target mRNA at the post-transcriptional level (18). miRNAs can bind to the 3'-UTR of the target gene mRNA by binding the RNA-induced silencing complex (19-21). In addition, miRNA activity can be impaired by lncRNA through sequestration, upregulating target gene expression (22). A previous study has demonstrated that miRNAs, such as miR-218, -203 and -146a, as well as miR-34 family (-34a/b/c) and let-7 family are potential biomarkers for the early diagnosis and treatment of COPD (23). Recently, miR-149-3p was demonstrated to be associated with smoking-induced COPD, and its downregulation increased inflammation in subjects with COPD via the Toll-like receptor-4/nuclear factor- $\kappa \mathrm{B}(\mathrm{NF}-\kappa \mathrm{B})$ signaling pathway (24). The principal regulator of inflammatory gene expression is $\mathrm{NF}-\kappa \mathrm{B}$ and its activity is controlled by I $\kappa \mathrm{B}$ proteins, whose stimulus-responsive degradation and re-synthesis provide for transient or dynamic regulation. Thus, NF- $\kappa \mathrm{B}$ signaling serves a major role in mediating inflammatory and innating immune responses (25). In lung tissues from patients with mild emphysema, the expression of miR-149-3p was decreased compared with tissues from patients with moderate emphysema (26). Thus, it was hypothesized that miR-149-3p may be involved in the $\mathrm{NF}-\kappa \mathrm{B}$ signaling pathway by regulating MEG3 expression.

The aim of the present study was to verify the role of IncRNA MEG3 in COPD, the underlying interaction between MEG3 and miR-149-3p and the effect of this interaction on cell proliferation and apoptosis in HEB cells.

\section{Materials and methods}

Preparation of cigarette smoke extract (CSE). CSE was collected as previously described (27). Briefly, the filters of two 3R4F research cigarettes from the University of Kentucky were removed, and the cigarettes were burned out in $5 \mathrm{~min}$. All the smoke produced by two cigarettes was transferred into a $50 \mathrm{ml}$ tube with $20 \mathrm{ml}$ sterile saline solution (Sinopharm Group Co., Ltd.), and the extract was filtered through a $0.22-\mu \mathrm{m}$ polytetrafluoroethylene filter (EMD Millipore).

Subjects and cell culture. A total of 66 subjects (16 women and 50 men; median age 58 years (range, 40-80 years);
18 non-smokers without COPD, 24 smokers without COPD and 28 smokers with COPD) were recruited at the Qinghai University Affiliated Hospital (Xining, China) between October 2018 and May 2019. Table I summarizes the patient characteristics and clinical features. Healthy subjects [forced expiratory volume in one second as a percentage of the predicted $(\mathrm{FEV} 1 \%) \geq 70 \%$ and forced expiratory volume in one second/forced vital capacity (FEV1/FVC) $\geq 70 \%$ ] (5) without chronic bronchitis and emphysema, and all patients with COPD were stable (FEV1/FVC $<70 \%)$ and had no exacerbations in the prior 6 months were enrolled. Peripheral blood samples were collected from the subjects, and maintained at $-80^{\circ} \mathrm{C}$. The present study was approved by the Ethics Committee of Qinghai University Affiliated Hospital, and all subjects provided written informed consent prior to participation in the study. The diagnosis of emphysema was made by the pathologist based on histological examination. All blood samples were maintained at $-80^{\circ} \mathrm{C}$ until processing of total RNA isolation.

The HBE cell line (HBE135-E6E7) was purchased from the American Type Culture Collection (cat. no. CRL-2741). HBE cells were cultured in RPMI-1640 (HyClone; GE Healthcare life Sciences) medium containing $10 \%$ fetal bovine serum (FBS; Gibco; Thermo Fisher Scientific, Inc.) with penicillin (100 U/ml 1/Streptomycin (100 $\mu \mathrm{g} / \mathrm{ml}$; Sigma-Aldrich; Merck $\mathrm{KGaA}$ ). The cells were cultured at $37^{\circ} \mathrm{C}$ in a $5 \% \mathrm{CO}_{2}$ incubator. Cells were pretreated at $37^{\circ} \mathrm{C}$ with $5 \% \mathrm{CSE}$ for $24 \mathrm{~h}$ before transfection and subsequent experiments.

Cell transfection. For transfection experiments, miR-149-3p mimics (miR-149-3p), miR-negative control (miR-NC) mimics, miR-149-3p inhibitor (anti-miR-149-3p), miR-NC inhibitor (anti-miR-NC), small interfering (si)RNA targeting MEG3 (si-MEG3\#1 and si-MEG3\#2 were used to screen the one with more significant suppressive effect on MEG3) and si-NC were obtained from Guangzhou RiboBio Co., Ltd. The MEG3 overexpression plasmid and empty vector was purchased from Hanbio Biotechnology Co., Ltd. In brief, the plasmids, miRNA mimics and miRNA inhibitors were transfected into $1 \times 10^{6} \mathrm{HBE} 135-\mathrm{E} 6 \mathrm{E} 7$ and pretreated at $37^{\circ} \mathrm{C}$ with $5 \% \mathrm{CSE}$ for 24 h using Lipofectamine ${ }^{\circledR} 3000$ (Invitrogen; Thermo Fisher Scientific Inc.) according to the manufacturer's instructions. Cells were cultured at $37^{\circ} \mathrm{C}$ with $5 \% \mathrm{CO}_{2}$ for $48 \mathrm{~h}$ after transfection. The sequences of miR-149-3p, miR-NC, anti-miR-149-3p, anti-miR-NC, si-MEG3\#1, si-MEG3\#2 are: miR-149-3p, 5'-AGGGAGGGACGGGGGCUGUGC-3'; miR-NC, 5'-UUC UCCGAACGUGUCACGUTT-3'; anti-miR-149-3p, 5'-GCA CAGCCCCCGUCCCUCCCU-3'; anti-miR-NC, 5'-CAGUAC UUUUGUGUAGUACAA-3'; si-MEG3\#1, 5'-AACAGCAAA UGGCACAGGAAGAGACGC-3'; and si-MEG3\#2, 5'-AUU GGAGGUGAGGAAGGAAAGCAGC-3'.

Reverse transcription-quantitative PCR (RT-qPCR). TRIzol ${ }^{\circledR}$ reagent (Invitrogen; Thermo Fisher Scientific Inc.) was used to extract the total RNA from the blood samples or HBE cells treated with various methods. The quality and quantity of total RNA were measured using the NanoDrop 2000 (Thermo Fisher Scientific Inc.). RNA reverse transcription was conducted using a GoScript Reverse Transcription System (Promega Corporation), and qPCR was performed using the 
Table I. Clinical and demographic characteristics of the study subjects $(n=66)$.

\begin{tabular}{lccc}
\hline Variables & Non-smokers & Smokers without COPD & Smokers with COPD \\
\hline Total subjects, $\mathrm{n}$ & 20 & 22 & 24 \\
Age, years & $53.4 \pm 3.6$ & $55.8 \pm 4.2$ & $62.1 \pm 2.5$ \\
Sex, n male/female & $12 / 8$ & $17 / 5$ & $21 / 3$ \\
BMI, kg/m & $22.4 \pm 1.3$ & $21.6 \pm 0.8$ & $22.1 \pm 1.5$ \\
FVC, \%pred. & $91.3 \pm 6.4$ & $80.5 \pm 10.2$ & $64.2 \pm 11.0$ \\
FEV1, \%pred. & $80.0 \pm 7.5$ & $66.3 \pm 8.1$ & $40.8 \pm 7.8$ \\
FEV1/FVC, \%pred. & $87.6 \pm 8.2$ & $82.4 \pm 7.2$ & $63.5 \pm 10.2$ \\
\hline
\end{tabular}

Values are expression as mean $\pm \mathrm{SD}$. COPD, chronic obstructive pulmonary disease; BMI, body mass index; FVC, forced vital capacity; $\%$ pred., percentage of predicted; $\mathrm{FEV}_{1}$, forced expiratory volume in $1 \mathrm{sec}$.

$\mathrm{SYBR}^{\circledR}$-Green I Supermix (Takara Biotechnology Co., Ltd.) according to the manufacturer's instructions. The thermocycling conditions were as follows: $95^{\circ} \mathrm{C}$ pre-denaturation for $3 \mathrm{~min}$, followed by 40 cycles of $95^{\circ} \mathrm{C}$ denaturation for $15 \mathrm{sec}$ and $60^{\circ} \mathrm{C}$ annealing for $1 \mathrm{~min}$. The relative expression of MEG3 or miR-149-3p was calculated using glyceraldehyde-3-phosphate dehydrogenase (GAPDH) or U6 small nuclear RNA (snRNA), respectively, as an internal control, and all data were calculated by the $2^{-\Delta \Delta \mathrm{Cq}}$ method (28) with three replicates. The primers were synthesized by Sangon Biotech Co, Ltd., and the primer sequences used were as follows: MEG3 forward, 5'-TCCATG CTGAGCTGCTGCCAAG-3' and reverse, 5'-AGTCGACAA AGACTGACACCC-3'; miR-149-3p forward, 5'-GAACCG GGATGGGAAGTGAC-3' and reverse, 5'-GCAAGCGGA ACTTCTAGCCT-3'; GAPDH forward, 5'-GACTCCACTCAC GGCAAATTCA-3' and reverse, 5'-TCGCTCCTGGAAGAT GGTGAT-3'; and U6 forward, 5'-CTCGCTTCGGCAGCA CA-3, and reverse 5'-AACGCTTCACGAATTTGCGT-3'.

Cell counting kit-8 (CCK-8) assay. HBE cell viability was assessed using the Cell Counting Kit-8 (CCK-8; Beyotime Institute of Biotechnology) according to the manufacturer's instructions. Following treatment with CSE, cells were seeded into 96-well plates at a density of $\sim 3,000$ cells/well. Untreated cells were used as a negative control. The cells were cultured at $37^{\circ} \mathrm{C}$ with $5 \% \mathrm{CO}_{2}$ for $48 \mathrm{~h}$ prior to the addition of $10 \mu \mathrm{l} \mathrm{CCK}-8$ reagent and incubated at $37^{\circ} \mathrm{C}$ for $2 \mathrm{~h}$. The optical density was detected by a microplate reader (Bio-Rad Laboratories Inc.) at $450 \mathrm{~nm}$.

Enzyme-linked immunosorbent assay (ELISA). Interleukin 6 (IL-6) is the most commonly assayed biomarkers to infer an underlying state of inflammation (29) and tumor necrosis factor- $\alpha$ (TNF- $\alpha$ ) plays an important pro-inflammatory role in COPD (30), thus IL- 6 and TNF- $\alpha$ were selected to detect the effect of MEG3 in inflammatory response. The expression of interleukin-6 (IL-6) and TNF- $\alpha$ were detected using Human IL- 6 and Human TNF- $\alpha$ ELISA Kits (cat. nos. ab46042 and ab181421, respectively; both Abcam) as previously described (31). The detailed procedure of ELISA was as follows: First, HBE cells induced by CSE and sterile saline solution were added into a simpleStep ELISA plate that had been coated with monoclonal antibody specific for IL-6 or TNF- $\alpha$ (from ELISA kit). Following incubation at $37^{\circ} \mathrm{C}$ for $30 \mathrm{~min}$, the plates were washed 3 times with PBS. Then, HBE cells were incubated with Human TNF- $\alpha$ Detector Antibody or Human IL-6 Detector Antibody (from the ELISA kits) at $37^{\circ} \mathrm{C}$ for $1 \mathrm{~h}$. Then $100 \mu \mathrm{l}$ of the TMB substrate was aded into each well and incubate for $30 \mathrm{~min}$ at room temperature in the dark. Finally, the Stop Solution was added into each well and the absorbance at $405 \mathrm{~nm}$ was measured by an ELISA instrument (Thermo Fisher Scientific Inc.) after a 20-min incubation with the chromogenic agent.

Apoptosis assay. To measure the apoptotic rates in HBE cells, the Annexin V-fluorescein isothiocyanate (FITC)/propidium iodide (PI) method was used, and the Annexin V-FITC/PI apoptosis detection kit was purchased from Beijing Solarbio Science \& Technology Co., Ltd.. According to the manufacturer's instructions, cells were harvested following culture for 2 days at $37^{\circ} \mathrm{C}$ with $5 \% \mathrm{CO}_{2}$ and resuspended in binding buffer. Subsequently, the cell suspension was mixed with Annexin V-FITC (1:20) and PI (1:20), followed by incubation at room temperature in the dark for $15 \mathrm{~min}$. Finally, the apoptotic cells were detected using a flow cytometer (BD Accuri ${ }^{\mathrm{TM}}$ C6; BD Biosciences) and FlowJo 10.2 (BD Biosciences) were used for analysis. The sum of apoptosis rate in right upper quadrant and right lower quadrant were considered as the cell apoptosis rate.

Western blotting. Total protein was extracted from HBE cells using RIPA lysis buffer (Beyotime Institute of Biotechnology) for $30 \mathrm{~min}$. A sodium dodecyl sulfate-polyacrylamide gel electrophoresis (SDS-PAGE) gel (10-15\%) was used to separate proteins and each lane had an equal amount of protein loaded in it $(\sim 30 \mu \mathrm{g})$. Subsequently, proteins were transferred to polyvinylidene fluoride membranes (EMD Millipore). 5\% BSA was used to dilute primary antibodies. Following blocking with $5 \%$ non-fat milk at room temperature for $1 \mathrm{~h}$, the membranes were incubated with primary antibodies against Bcl-2 (1:1,000; cat. no. ab32124; Abcam), caspase-3 (1:1,000; cat. no. ab2302; Abcam), caspase-9 (1:1,000; cat. no. ab2324; Abcam), p65 (1:1,000; cat. no. ab16502; Abcam), phospho S536-p65 (1:1,000; cat. no. ab86299; Abcam), IкB $\alpha$ (1:1,000; cat. no. ab95338; Abcam); phospho-S36-IкB $\alpha$ (1:1,000; cat. no. ab133462; Abcam) or GAPDH (1:1,000; cat. no. ab37168; Abcam), 

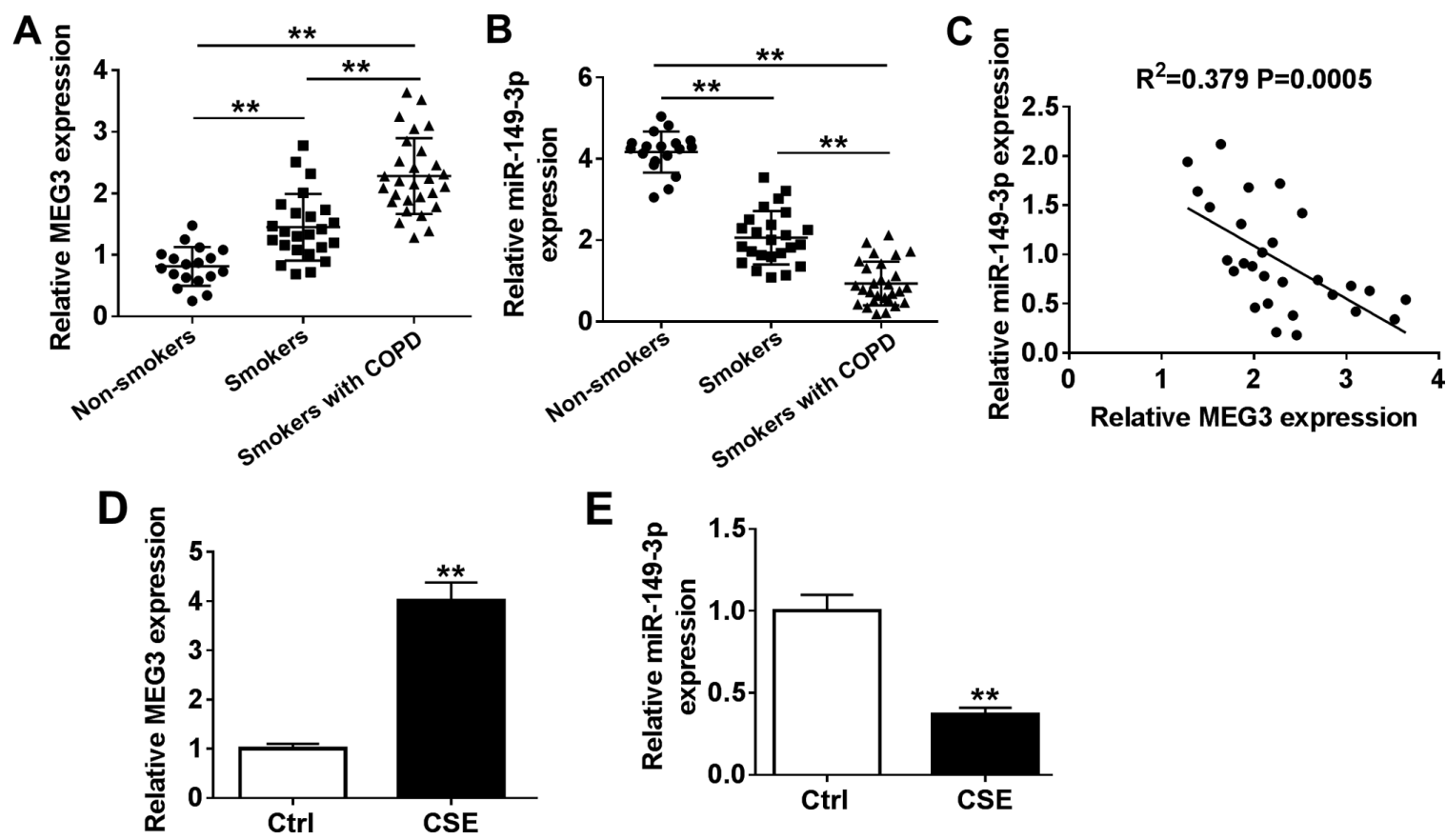

Figure 1. Relative mRNA expression levels of long non-coding RNA MEG3 and miR-149-3p in blood samples from patients with COPD and CSE-induced cells. (A and B) mRNA expression levels of MEG3 and miR-149-3p were measured by RT-qPCR in non-smokers, smokers and smokers with COPD. (C) Correlation analysis of MEG3 and miR-149-3p mRNA expression levels in smokers with COPD. (D and E) Relative mRNA expression levels of MEG3 and miR-149-3p were detected in untreated (Ctrl) and CSE-induced human bronchial epithelial cells using RT-qPCR. ${ }^{* *} \mathrm{P}<0.01$. MEG3, maternally expressed gene 3; RT-q, reverse-transcription quantitative; miR, microRNA; CSE, cigarette smoke extract; COPD, chronic obstructive pulmonary disease.

which HRP-conjugated secondary antibody Goat Anti-Rabbit IgG H\&L (1:20,000; cat. no. ab97051; Abcam) was used to probe the proteins on the membranes at room temperature for $1 \mathrm{~h}$. Finally, protein bands were visualized using a commercial enhanced chemiluminescence chromogenic substrate (Beyotime Institute of Biotechnology), and the densitometry and quantity of the protein was analyzed using Quantity One 1-D Analysis software (v4.6.6; Bio-Rad Laboratories Inc.).

Dual luciferase reporter assay. -0.616; $\mathrm{P}<0.001$; Fig. 1C. In addition, the expression of MEG3 was significantly upregulated in CSE-induced HBE cells compared with that in the control cells, whereas miR-149-3p expression was reduced (Fig. 1D and E).

MEG3 inhibits cell proliferation, induces apoptosis and regulates inflammatory cytokine expression in CSE-induced HBE cells. To explore the role of MEG3 in COPD, HBE cells were used for further investigation. RT-qPCR was performed in untreated and CSE-induced HBE cells. The expression of MEG3 was elevated in CSE-induced HBE cells compared with untreated HBE cells, but reduced in CSE-induced HBE cells transfected with si-MEG3 compared with HBE cells transfected with si-NC (Fig. 2A). The CCK-8 assay revealed that cell proliferation in CSE-induced HBE cells was prompted by the knockdown of MEG3 (Fig. 2B). In addition, the levels of IL- 6 and TNF- $\alpha$ were measured by ELISA, and the results demonstrated that the levels of the two cytokines was significantly reduced in CSE-induced HBE cells transfected with si-MEG3 compared with CSE-induced HBE cells transfected with si-NC (Fig. 2C and D). The apoptotic rate was markedly decreased in CSE-induced HBE cells transfected with si-MEG3 compared with that in the CSE-treated si-NC group (Fig. 2E). Western blotting was used to determine the protein expression levels of Bcl-2, cleaved-caspase-3 and cleaved-caspase-9; compared with the si-NC group, knockdown of MEG3 enhanced the protein expression of Bcl-2 and inhibited that of cleaved-caspase-3/pro-caspase-3 and cleaved-caspase-9/pro-caspase-9 in CSE-induced HBE cells (Fig. 2F). In conclusion, MEG3 knockdown enhanced cell proliferation, inhibited apoptosis and regulated inflammatory cytokine levels in CSE-induced HBE cells.

MEG3 targets miR-149-3p to downregulate its expression. The DIANA online tool was used to predict the binding site of MEG3 and miR-149-3p. It was identified that MEG3 may bind miR-149-3p. It was also observed that there was a potential binding site for miR-149-3p in MEG3 and certain bases were changed to obtain MEG3 mutant sequence (Fig. 3A). To verify this prediction, MEG3-WT and MEG3-MUT vectors with luciferase reporter were constructed and co-transfected into cells with miR-149-3p. The transfection efficiency of miR-149-3p mimics was first verified (Fig. S1A). The luciferase activity of MEG3-WT in HEB cells significantly declined in the presence of miR-149-3p mimics while HEB cells co-transfected with MEG3-MUT and miR-149-3p did not exhibit a significant difference (Fig. 3B). However, there were no notable changes in the luciferase activity of MEG3-MUT in the presence of miR-149-3p mimics (Fig. 3B). To further explore the relationship between MEG3 and miR-149-3p, overexpression and knockdown MEG3 plasmids were transfected into HBE cells, and RT-qPCR was used to 
A

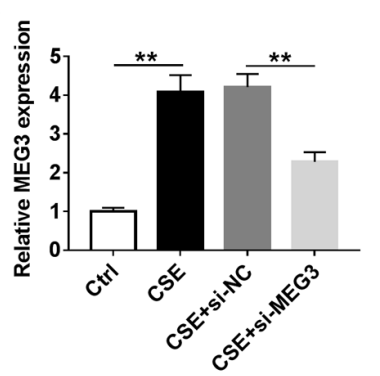

B

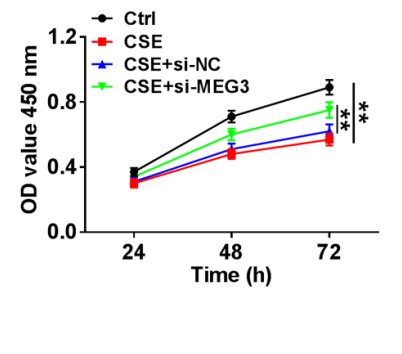

C

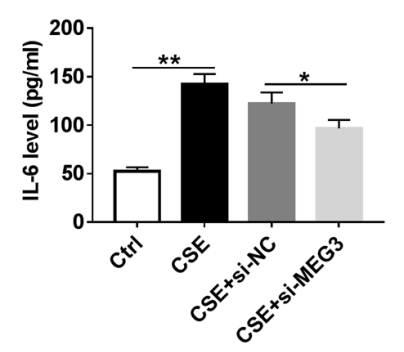

D

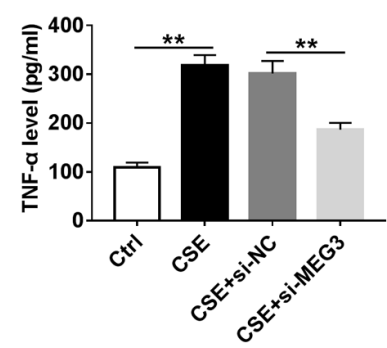

E

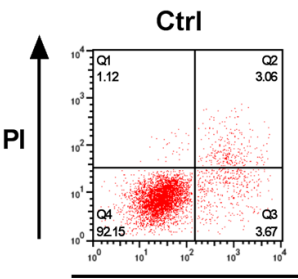

CSE

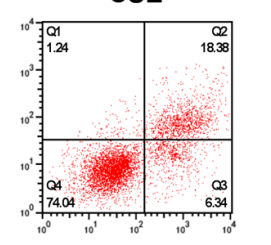

CSE+si-NC

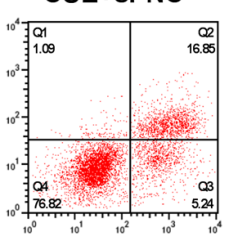

CSE+si-MEG3

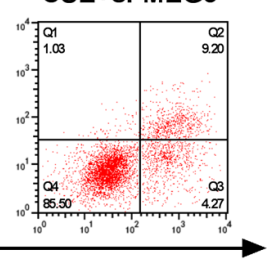

Annexin V-FITC

$\mathbf{F}$

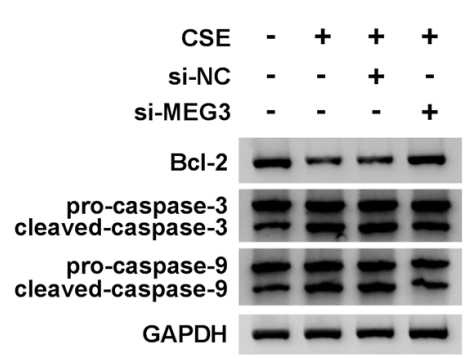

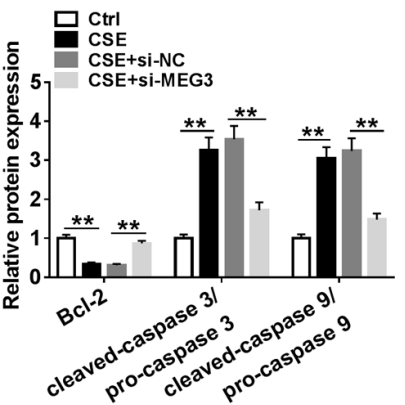

Figure 2. Effects of MEG3 knockdown on cell proliferation, apoptosis and inflammatory cytokine expression in CSE-induced HBE cells. Human bronchial epithelial cells were treated with control (untreated HBE cells), CSE, CSE + si-NC or CSE + si-MEG3. (A) mRNA expression of MEG3 in treated HBE cells was measured using reverse transcription-quantitative PCR. (B) Cell proliferation was examined by the Cell Counting Kit-8 assay. (C and D) The levels of IL-6 and TNF- $\alpha$ were detected using ELISA. (E) Apoptosis was analyzed by flow cytometry. (F) Protein expression levels of Bcl-2, caspase-3 and caspase-9 were determined by western blotting. ${ }^{* *} \mathrm{P}<0.01$. MEG3, maternally expressed gene 3 ; miR, microRNA; CSE, cigarette smoke extract; OD, optical density; PI, propidium iodide; NC, negative control; IL, interleukin; TNF, tumor necrosis factor; si, small interfering RNA.

verify the up- and downregulation of MEG3 expression (Fig. 3C and D). Subsequently, the level of miR-149-3p was measured in cells transfected with MEG3 or si-MEG3; the results demonstrated that the expression of miR-149-3p was significantly reduced in HBE cells overexpressing MEG3, but increased in cells transfected with si-MEG3 compared with the respective negative controls (Fig. 3E and F). These results suggested that miR-149-3p was a target of MEG3 and was downregulated by MEG3.

MEG3 enhances cell proliferation and reduces the apoptotic rate in CSE-induced HBE cells by targeting $m i R-149-3 p$. To verify the effects of MEG3 on regulating cell proliferation and apoptosis, anti-miR-NC, miR-149-3p inhibitor or si-MEG3 were transfected into CSE-induced HBE cells. The transfection efficiency of the miR-149-3p inhibitor was verified and presented in Fig. S1B. RT-qPCR was performed to detect the expression of miR-149-3p in CSE-treated HBE cells. The results revealed that miR-149-3p expression was significantly decreased in cells treated with the miR-149-3p inhibitor compared with that in the
miR-NC group; similarly, the expression of miR-149-3p was upregulated in CSE-induced HBE cells with MEG3 knockdown, but it was reduced when co-treated with miR-149-3p inhibitor (Fig. 4A). Next, the proliferation of HBE cells was analyzed using the CCK- 8 assay. Compared with untreated CSE-induced HBE cells, miR-149-3p inhibitor treated group reduced the proliferation of CSE-induced HBE cells obviously (Fig. 4B). When CSE-induced HBE cells were co-transfected with si-MEG3 and miR-149-3p, cell proliferative ability was also decreased following 72 -h culture compared with CSE-induced HBE cells co-transfected with si-MEG3 and miR-NC (Fig. 4B). Apoptosis of HBE cells was measured using flow cytometry, which revealed that apoptosis was enhanced when miR-149-3p was inhibited in GSE-induced HBE cells, and this apoptosis inducing effect was reversed by co-transfected group (miR-149-3p inhibitor and si-MEG3) (Fig. 4C and D). Western blotting was performed to detect the expression of the apoptosis-related proteins Bcl-2, cleaved-caspase-3 and cleaved-caspase-9. The results demonstrated that the expression of Bcl-2 was downregulated, whereas cleaved-caspase-3/pro-caspase-3 
A

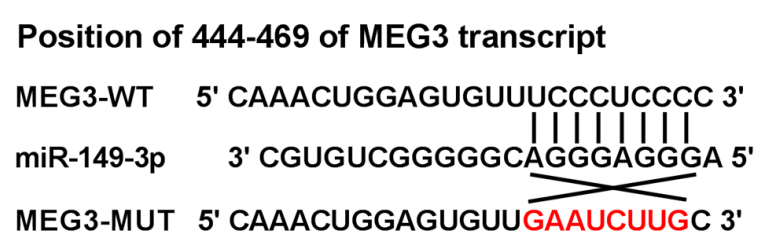

MEG3-MUT 5' CAAACUGGAGUGUUGAAUCUUGC 3 '
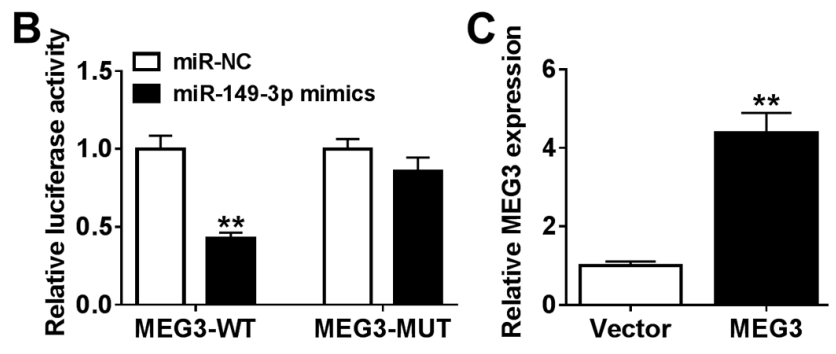

D

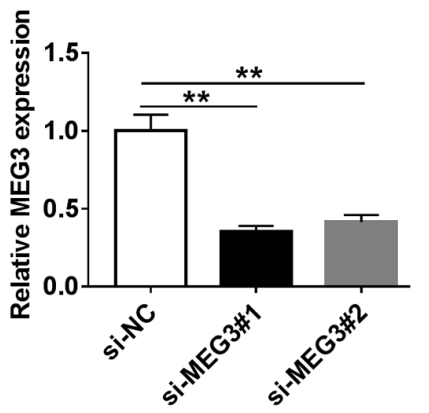

E

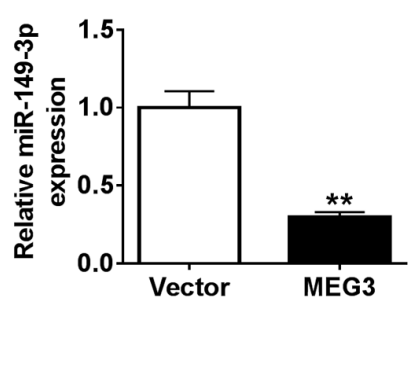

$\mathbf{F}$

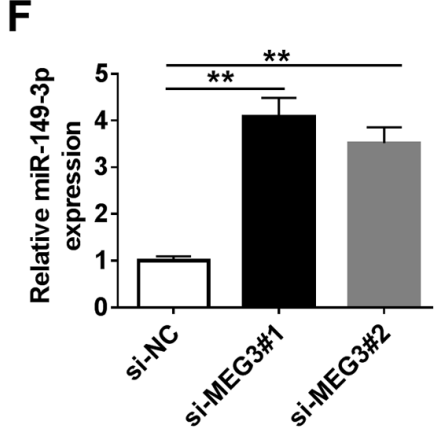

Figure 3. Relationship between MEG3 and miR-149-3p. (A) The DIANNA online tool predicted the putative binding sites of MEG3 and miR-149-3p and MUT sequence of MEG3 is shown in red. (B) Luciferase activity was evaluated in HBE cells co-transfected with MEG3-WT or MEG3-MUT and miR-NC or miR-149-3p mimics. (C and E) The expression of MEG3 or miR-149-3p was examined in HBE cells transfected with empty vector and MEG3. (D and F) Levels of MEG3 or miR-149-3p were measured in HBE cells transfected with si-NC, si-MEG3\#1, and si-MEG3\#2. ** P<0.01. MEG3, maternally expressed gene 3; miR, microRNA; CSE, cigarette smoke extract; HBE, human bronchial epithelial; NC, negative control; si, small interfering RNA; WT, wild-type; MUT, mutant.

and cleaved-caspase-9/pro-caspase-9 were upregulated in CSE-induced cells transfected with si-MEG3 and miR-149-3p inhibitor compared with CSE-induced cells transfected with si-MEG3 and miR-NC (Fig. 4E). An ELISA assay demonstrated that the levels of IL- 6 and TNF- $\alpha$, which are important inflammatory cytokines, were increased by the simultaneous knockdown of MEG3 and miR-149-3p compared with CSE-induced cells transfected with si-MEG3 and miR-NC (Fig. 4F and G). Taken together, these results demonstrated that miR-149-3p partly reversed the regulating effect of MEG3 on cell proliferation, apoptosis and inflammatory cytokine expression in CSE-induced HBE cells.

$M E G 3$ regulates the $N F-\kappa B$ signaling pathway in $C S E$-induced HBE cells by targeting miR-149-3p. To elucidate the molecular mechanism of MEG3 and miR-149-3p

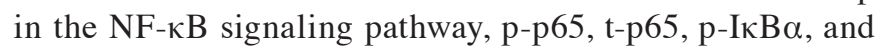
$\mathrm{t}-\mathrm{I} \kappa \mathrm{B} \alpha$, which are involved in the $\mathrm{NF}-\kappa \mathrm{B}$ pathway, were selected for evaluation using western blotting. CSE-induced HBE cells were co-transfected with miR-149-3p inhibitor or/and si-MEG3 (Fig. 5). The ratio of p-p65/t-p65 and $\mathrm{p}-\mathrm{I} \kappa \mathrm{B} \alpha / \mathrm{t}-\mathrm{I} \kappa \mathrm{B} \alpha$ was increased when the expression of miR-149-3p was inhibited by miR-149-3p inhibitor, compared with the negative control group; however, it would be reversed when MEG3 was downregulated with miR-149-3p inhibitor in CSE-induced HBE cells (Fig. 5). These results suggested that MEG3 regulates the $N F-\kappa B$ signaling pathway by targeting miR-149-3p.

\section{Discussion}

The aim of the present study was to investigate the potential functions and the underlying molecular mechanism of lncRNA
MEG3 in COPD. The results of the present study indicated that MEG3 was highly expressed in blood samples from subjects with COPD, and MEG3 knockdown promoted cell proliferation and inhibited apoptosis in HBE cells. In addition, miR-149-3p mRNA expression was downregulated in blood samples from subjects that smokers with COPD compared with non-smokers or smokers without COPD and demonstrated an inverse correlation with MEG3. The results of the present study also revealed that miR-149-3p was a target of MEG3 and regulated the expression of apoptotic proteins, such as bcl-2, cleaved caspase 3 and cleaved caspase 9, and inflammatory cytokines, such as IL- 6 and TNF- $\alpha$. In summary, the present study demonstrated that the effects of cell proliferation and apoptosis induced by MEG3 could be restored by miR-149-3p mimics in CSE induced HBE cells, which was involved in the $\mathrm{NF}-\kappa \mathrm{B}$ signaling pathway. The results of the present study provide new evidence for the elucidation and better understanding of the molecular mechanism of MEG3 in COPD.

lncRNAs have been reported to participate in various biological and physiological processes, such as epigenetic regulation, maintaining cell biological and morphological characteristics and transcriptional regulation (32). IncRNAs, for example, TUG1 have been demonstrated to regulate tumor growth and the development of lung cancer (33). Silencing MEG3 suppresses HBE cell apoptosis and autophagy induced by fine particulate matter (PM 2.5); however, the molecular basis of the effects of lncRNA in subjects with COPD remains unclear (17). Compared with smokers and non-smokers, MEG3 expression was significantly elevated in blood samples from subjects with COPD in the present study, which was consistent with the results of a previous study (16). In the present study, knockdown of MEG3 triggered cell 
A

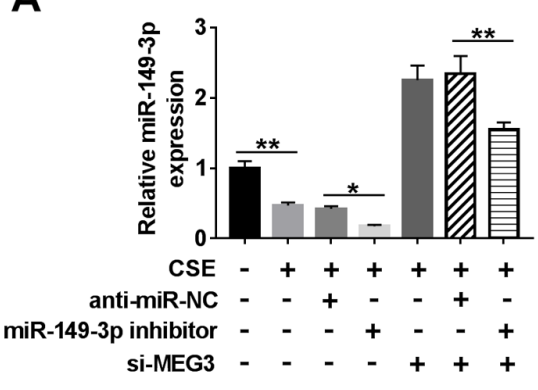

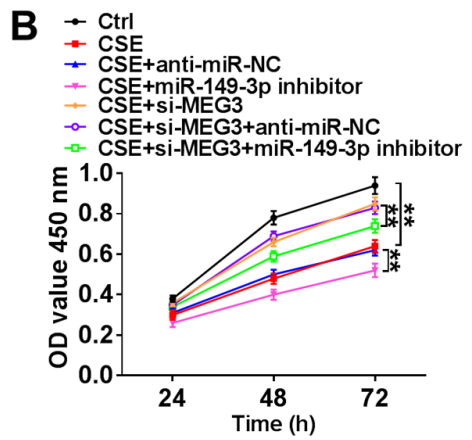

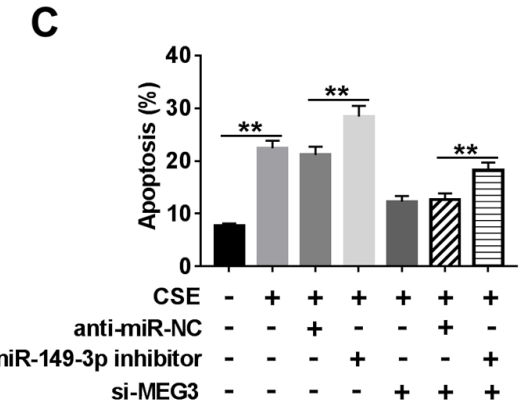

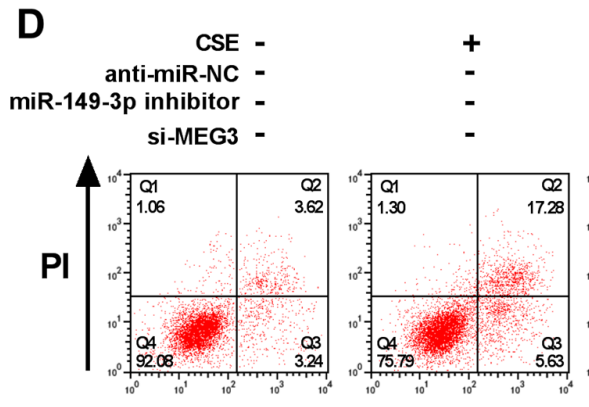
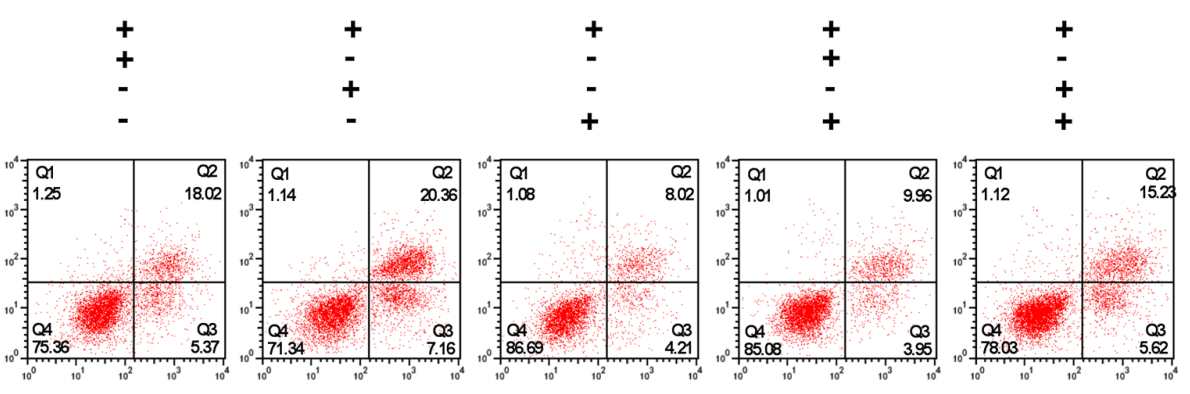

Annexin V-FITC

E

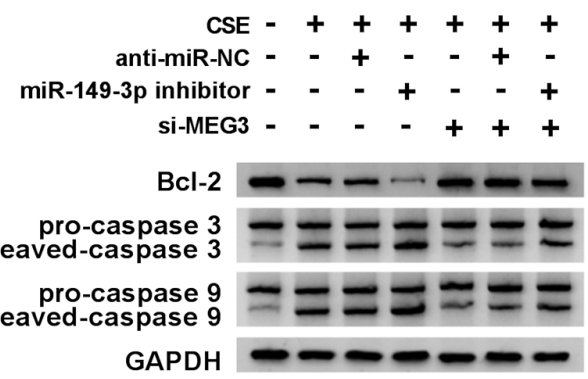

$\mathbf{F}$

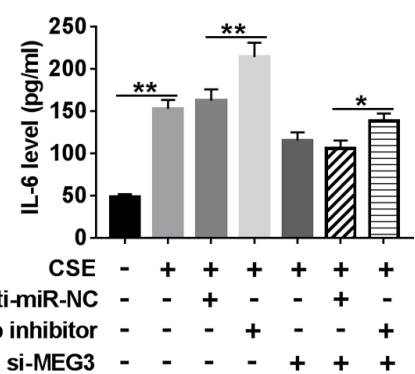

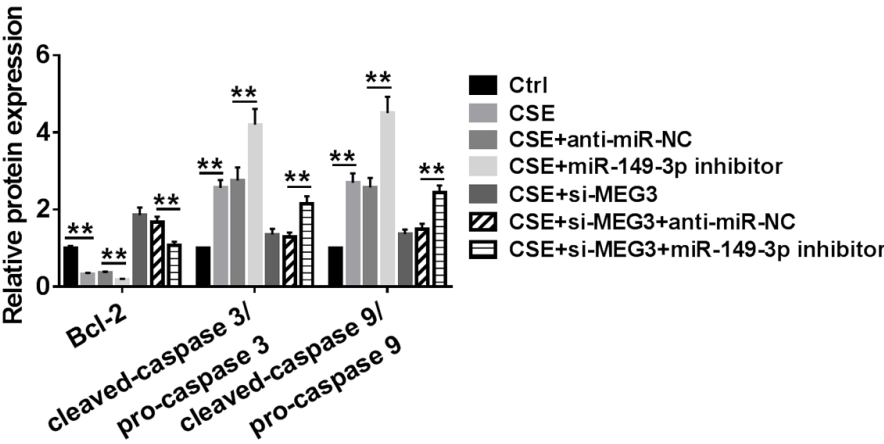

G

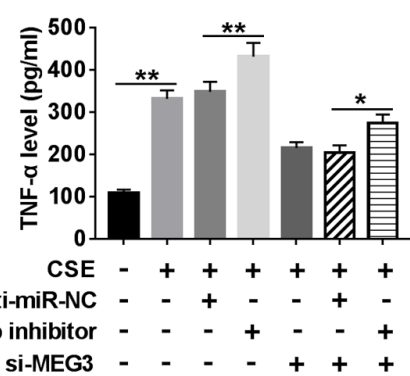

Figure 4. MEG3 regulatory effects on miR-149-3p-mediated HBE cell proliferation, apoptosis and inflammatory cytokine expression. HBE cells were treated with control (untreated HBE cells), CSE, CSE + anti-miR-NC, CSE + miR-149-3p inhibitor, CSE + si-MEG3, CSE + si-MEG3 + anti-miR-NC or CSE + si-MEG3 + miR-149-3p inhibitor. (A) The expression of miR-149-3p was determined using reverse-transcription quantitative PCR in treated HBE cells. (B) Cell proliferation of treated HBE cells was determined using the Cell Counting Kit-8 assay. (C and D) Apoptosis analyzed by flow cytometry. (E) Protein levels of Bcl-2, caspase-3 and caspase-9 were measured by western blotting. (F) ELISA was used to measure the levels of IL-6 and (G) TNF- $\alpha$. "** $\mathrm{P}<0.01$. MEG3, maternally expressed gene 3; miR, microRNA; CSE, cigarette smoke extract; OD, optical density; PI, propidium iodide; NC, negative control; IL, interleukin; TNF, tumor necrosis factor; si, small interfering RNA.

proliferation and inhibited apoptosis in CSE-induced HBE cells. In addition, the rescue experiments of the present study demonstrated that downregulation of miR-149-3p rescued the effects of MEG3 in CSE-induced HBE cells. These results indicated that MEG3 may serve a crucial role in COPD by binding miR-149-3p.
Research have indicated that miRNAs may be sponged by lncRNAs, which have been identified as competing endogenous RNAs (34). In the present study, a relationship between MEG3 and miR-149-3p was identified. RT-qPCR was conducted to detect the expression of miR-149-3p in peripheral blood samples from non-smokers without COPD, smokers 

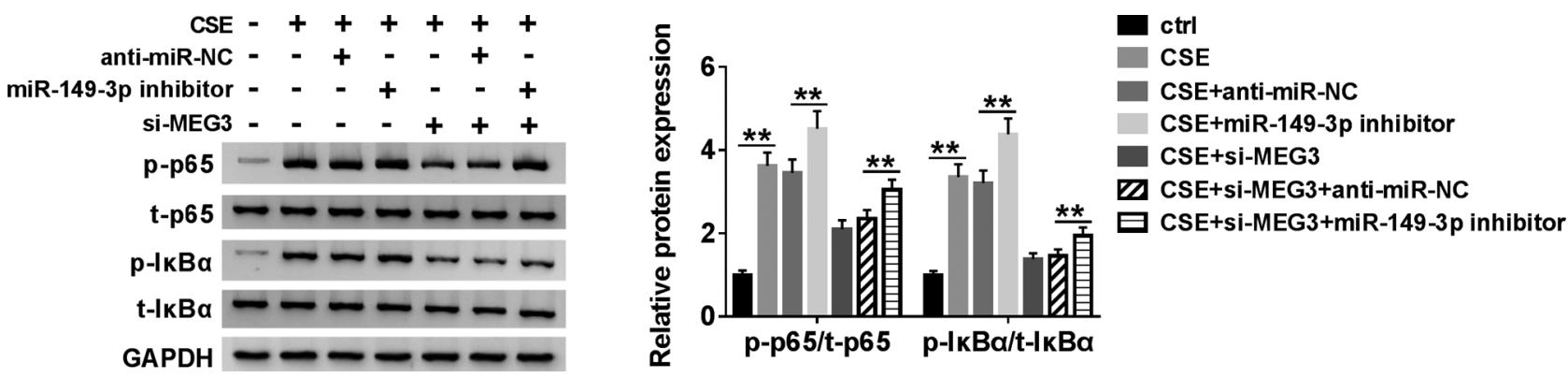

Figure 5. MEG3 regulates the NF- $\mathrm{KB}$ signaling pathway by targeting miR-149-3p. HBE cells were treated with control (untreated HBE cells), CSE, CSE + anti-miR-NC, CSE + miR-149-3p inhibitor, CSE + si-MEG3, CSE + si-MEG3 + anti-miR-NC or CSE + si-MEG3 + miR-149-3p inhibitor. The protein expression levels of p-p65, t-p65, p-IкB $\alpha, \mathrm{p}-\mathrm{I} \kappa \mathrm{B} \alpha$ in treated cells were determined by western blotting. ${ }^{*} \mathrm{P}<0.01$. MEG3, maternally expressed gene 3; miR, microRNA; CSE, cigarette smoke extract; NC, negative control; p, phosphorylated; t, total; si, small interfering RNA.

without COPD and smokers with COPD and HEB cells treated with CSE or not. Moreover, the low expression of miR-149-3p in smokers with COPD was regulated by MEG3.

Previous studies have demonstrated that the NF- $\kappa \mathrm{B}$ signaling pathway serves a crucial role in the development of lipopolysaccharide-induced acute lung injury (35) and in cell inflammatory response $(36,37)$. In addition, $\mathrm{Bcl}-2$ has been demonstrated to be an important biomarker for inflammatory function in the NF- $\mathrm{KB}$ signaling pathway (38). In the present study, the protein expression levels of Bcl-2 were measured in HBE cells in addition to those of cleaved-caspase-3 and cleaved-caspase-9, which are associated with cell apoptosis (39); the level of Bcl-2 was increased in the presence of si-MEG3 in CSE-induced HBE cells compared with CSE-induced HBE cells transfected with si-NC. However, an opposite trend was observed for cleaved-caspase-3 and cleaved-caspase-9. In addition, the inhibition of miR-149-3p repressed the level of Bcl-2 when cells were co-transfected with si-MEG3. All the aforementioned results of the present study demonstrated that MEG3 regulated the inflammatory response by targeting miR-149-3p.

The present study provided new evidence to elucidate the function of lncRNA MEG3 in blood samples from subjects with COPD. miR-149-3p mimics reversed the effects of MEG3 on cell proliferation, apoptosis and inflammatory cytokine expression. In addition, in the present study, MEG3 regulated the NF- $\kappa \mathrm{B}$ signaling pathway in HBE cells by targeting miR-149-3p. It has been reported that miR-149-3p is involved in the Akt1 signaling pathway in pancreatic cancer (40) and in the p53-signaling pathway in non-small-cell lung cancer (41). Future studies should focus on whether the regulatory effects of MEG3/miR-149-3p in COPD may be mediated by other signaling pathways. Although the results of the present study have revealed the potential role of MEG3 and miR-149-3p in vitro, in vivo studies using animal models are essential for gaining a deeper understanding of the molecular mechanism of COPD. Smoking is the strongest, but not the only risk factor for COPD, and further studies are required to explore the regulatory mechanisms of other pathogenic factors of COPD.

In conclusion, in the present study, upregulation of MEG3 and downregulation of miR-149-3p expression was observed in blood samples of smokers with COPD and in CSE-induced HBE cells. In addition, inhibition of miR-149-3p reversed the MEG3 knockdown-mediated effects on cell proliferation and apoptosis in CSE-induced HBE cells. MEG3 was demonstrated to regulate the NF- $\kappa \mathrm{B}$ signaling pathway in CSE-induced HBE cells by targeting miR-149-3p. Thus, the results of the present study demonstrated a role of the MEG3/miR-149-3p axis in CSE-induced HBE cells and provided new insight into the molecular basis of COPD, which requires further verification.

\section{Acknowledgements}

Not applicable.

\section{Funding}

No funding was received.

\section{Availability of data and materials}

The datasets used and/or analyzed during the current study are available from the corresponding author on reasonable request.

\section{Authors' contributions}

SZ, ZL and JJ conceived and designed the study. ZL and HG carried out the experiments. JS performed data mining, acquisition and analysis of data. SZ, YK and JJ wrote and approved the final manuscript. All authors have read and approved the manuscript.

\section{Ethics approval and consent to participate}

The present study was approved by the Ethics Committee of Qinghai University Affiliated Hospital (Xining, China), and all subjects provided written informed consent prior to participation in the study.

\section{Patient consent for publication}

Not applicable.

\section{Competing interests}

The authors declare that they have no competing interests. 


\section{References}

1. Centers for Disease Control and Prevention (CDC): Chronic obstructive pulmonary disease among adults-United States, 2011. MMWR Morb Mortal Wkly Rep 61: 938-943, 2012.

2. Incalzi RA, Scarlata S, Pennazza G, Santonico M and Pedone C: Chronic obstructive pulmonary disease in the elderly. Eur J Intern Med 25: 320-328, 2014.

3. Adeloye D, Chua S, Lee C, Basquill C, Papana A, Theodoratou E, Nair H, Gasevic D, Sridhar D, Campbell H, et al: Global and regional estimates of COPD prevalence: Systematic review and meta-analysis. J Glob Health 5: 020415, 2015.

4. GBD 2015 Chronic Respiratory Disease Collaborators: Global, regional, and national deaths, prevalence, disability-adjusted life years, and years lived with disability for chronic obstructive pulmonary disease and asthma, 1990-2015: A systematic analysis for the global burden of disease study 2015. Lancet Respir Med 5: 691-706, 2017.

5. Vogelmeier CF, Criner GJ, Martinez FJ, Anzueto A, Barnes PJ, Bourbeau J, Celli BR, Chen R, Decramer M, Fabbri LM, et al: Global strategy for the diagnosis, management, and prevention of chronic obstructive lung disease 2017 report. GOLD executive summary. Am J Respir Crit Care Med 195: 557-582, 2017.

6. Lamprecht B, McBurnie MA, Vollmer WM, Gudmundsson G, Welte T, Nizankowska-Mogilnicka E, Studnicka M, Bateman E, Anto JM, Burney P, et al: COPD in never smokers: Results from the population-based burden of obstructive lung disease study. Chest 139: 752-763, 2011

7. Zarghami M, Taghizadeh F, Sharifpour A and Alipour A: Efficacy of smoking cessation on stress, anxiety, and depression in smokers with chronic obstructive pulmonary disease: A randomized controlled clinical trial. Addict Health 10: 137-147, 2018.

8. Bhan A, Soleimani M and Mandal SS: Long noncoding RNA (lncRNA): Functions in health and disease. Gene Regulation, Epigenetics and Hormone Signaling, 2017.

9. Hon KW, Abu N, Ab Mutalib NS and Jamal R: MiRNAs and lncRNAs as predictive biomarkers of response to FOLFOX therapy in colorectal cancer. Front Pharmacol 9: 846, 2018.

10. Heward JA and Lindsay MA: Long non-coding RNAs in the regulation of the immune response. Trends Immunol 35: 408-419, 2014.

11. Zheng M, Hong W, Gao M, Yi E, Zhang J, Hao B, Liang C, Li X, Li C, Ye X, et al: Long noncoding RNA COPDA1 promotes airway smooth muscle cell proliferation in chronic obstructive pulmonary disease. Am J Respir Cell Mol Biol 61: 584-596, 2019

12. Qian Y, Mao ZD, Shi YJ, Liu ZG, Cao Q and Zhang Q: Comprehensive analysis of miRNA-mRNA-lncRNA networks in non-smoking and smoking patients with chronic obstructive pulmonary disease. Cell Physiol Biochem 50: 1140-1153, 2018.

13. Ge J, Geng S and Jiang H: Long noncoding RNAs antisense noncoding RNA in the INK4 locus (ANRIL) correlates with lower acute exacerbation risk, decreased inflammatory cytokines, and mild GOLD stage in patients with chronic obstructive pulmonary disease. J Clin Lab Anal 33: e22678, 2019.

14. Josipovic I, Pflüger B, Fork C, Vasconez AE, Oo JA, Hitzel J, Seredinski S, Gamen E, Heringdorf DMZ, Chen W, et al: Long noncoding RNA LISPR1 is required for S1P signaling and endothelial cell function. J Mol Cell Cardiol 116: 57-68, 2018.

15. Liu W, Luo M, Zou L, Liu X, Wang R, Tao H, Wu D, Zhang W, Luo $Q$ and Zhao Y: uNK cell-derived TGF- $\beta 1$ regulates the long noncoding RNA MEG3 to control vascular smooth muscle cell migration and apoptosis in spiral artery remodeling. J Cell Biochem 120: 15997-16007, 2019.

16. Tang W, Shen Z, Guo J and Sun S: Screening of long non-coding RNA and TUG1 inhibits proliferation with TGF- $\beta$ induction in patients with COPD. Int J Chron Obstruct Pulmon Dis 11: 2951-2964, 2016.

17. Li X, Zheng M, Pu J, Zhou Y, Hong W, Fu X, Peng Y, Zhou W, Pan H, Li B and Ran P: Identification of abnormally expressed lncRNAs induced by PM2.5 in human bronchial epithelial cells. Biosci Rep 38: BSR20171577, 2018.

18. Trionfini P and Benigni A: MicroRNAs as master regulators of glomerular function in health and disease. J Am Soc Nephrol 28: 1686-1696, 2017

19. Bartel DP: Metazoan MicroRNAs. Cell 173: 20-51, 2018.

20. Li D, Li YP, Li YX, Zhu XH, Du XG, Zhou M, Li WB and Deng HY: Effect of regulatory network of exosomes and microRNAs on neurodegenerative diseases. Chin Med J (Engl) 131: 2216-2225, 2018.
21. Karatas OF, Oner M, Abay A and Diyapoglu A: MicroRNAs in human tongue squamous cell carcinoma: From pathogenesis to therapeutic implications. Oral Oncol 67: 124-130, 2017.

22. Thomson DW and Dinger ME: Endogenous microRNA sponges: Evidence and controversy. Nat Rev Genet 17: 272-283, 2016.

23. Huang X, Zhu Z, Guo X and Kong X: The roles of microRNAs in the pathogenesis of chronic obstructive pulmonary disease. Int Immunopharmacol 67: 335-347, 2019.

24. Shen W, Liu J, Zhao G, Fan M, Song G, Zhang Y, Weng Z and Zhang Y: Repression of Toll-like receptor- 4 by microRNA-149-3p is associated with smoking-related COPD. Int J Chron Obstruct Pulmon Dis 12: 705-715, 2017.

25. Adelaja A and Hoffmann A: Signaling crosstalk mechanisms that may fine-tune pathogen-responsive NFKB. Front Immunol 10: 433, 2019.

26. Savarimuthu Francis SM, Davidson MR, Tan ME, Wright CM, Clarke BE, Duhig EE, Bowman RV, Hayward NK, Fong KM and Yang IA: MicroRNA-34c is associated with emphysema severity and modulates SERPINE1 expression. BMC Genomics 15: 88, 2014.

27. Wang J, Li Q, Xie J and Xu Y: Cigarette smoke inhibits BAFF expression and mucosal immunoglobulin A responses in the lung during influenza virus infection. Respir Res 16: 37, 2015.

28. Livak KJ and Schmittgen TD: Analysis of relative gene expression data using real-time quantitative PCR and the 2(-Delta Delta C(T)) method. Methods 25: 402-408, 2001.

29. Del Giudice M and Gangestad SW: Rethinking IL-6 and CRP: Why they are more than inflammatory biomarkers, and why it matters. Brain Behav Immun 70: 61-75, 2018

30. Antoniu SA, Mihaltan F and Ulmeanu R: Anti-TNF-alpha therapies in chronic obstructive pulmonary diseases. Expert Opin Investig Drugs 17: 1203-1211, 2008.

31. Takahashi E, Kataoka K, Indalao IL, Konoha K, Fujii K, Chida J, Mizuno D, Fujihashi K and Kido H: Oral clarithromycin enhances airway immunoglobulin $\mathrm{A}(\operatorname{Ig} \mathrm{A})$ immunity through induction of IgA class switching recombination and B-cell-activating factor of the tumor necrosis factor family molecule on mucosal dendritic cells in mice infected with influenza A virus. J Virol 86: 10924-10934, 2012.

32. Lin C and Yang L: Long noncoding RNA in cancer: Wiring signaling circuitry. Trends Cell Biol 28: 287-301, 2018.

33. Beermann J, Piccoli MT, Viereck J and Thum T: Non-coding RNAs in development and disease: Background, mechanisms, and therapeutic approaches. Physiol Rev 96: 1297-1325, 2016.

34. Zhuan B, Lu Y, Chen Q, Zhao X, Li P, Yuan Q and Yang Z: Overexpression of the long noncoding RNA TRHDE-AS1 inhibits the progression of lung cancer via the miRNA-103/KLF4 axis. J Cell Biochem 120: 17616-17624, 2019.

35. Zhu G, Xin X, Liu Y, Huang Y, Li K and Wu C: Geraniin attenuates LPS-induced acute lung injury via inhibiting NF- $\kappa \mathrm{B}$ and activating Nrf2 signaling pathways. Oncotarget 8: 22835-22841, 2017.

36. Jin LY, Li CF, Zhu GF, Wu CT, Wang J and Yan SF: Effect of siRNA against NF- $\kappa \mathrm{B}$ on sepsis-induced acute lung injury in a mouse model. Mol Med Rep 10: 631-637, 2014.

37. Wu C, Zhao J, Zhu G, Huang Y and Jin L: SiRNA directed against $\mathrm{NF}-\kappa \mathrm{B}$ inhibits mononuclear macrophage cells releasing proinflammatory cytokines in vitro. Mol Med Rep 16: 9060-9066, 2017.

38. Badrichani AZ, Stroka DM, Bilbao G, Curiel DT, Bach FH and Ferran C: Bcl-2 and Bcl-XL serve an anti-inflammatory function in endothelial cells through inhibition of NF-kappaB. J Clin Invest 103: 543-553, 1999.

39. Kopeina GS, Prokhorova EA, Lavrik IN and Zhivotovsky B: Alterations in the nucleocytoplasmic transport in apoptosis: Caspases lead the way. Cell Prolif 51: e12467, 2018.

40. Si L, Xu L, Yin L, Qi Y, Han X, Xu Y, Zhao Y, Liu K and Peng J: Potent effects of dioscin against pancreatic cancer via miR-149-3P-mediated inhibition of the Akt1 signalling pathway. Br J Pharmacol 174: 553-568, 2017.

41. Zou A, Liu X, Mai Z, Zhang J, Liu Z, Huang Q, Wu A and Zhou C: LINC00472 acts as a tumor suppressor in NSCLC through KLLN-mediated p53-signaling pathway via MicroRNA-149-3p and MicroRNA-4270. Mol Ther Nucleic Acids 17: 563-577, 2019.

This work is licensed under a Creative Commons Attribution-NonCommercial-NoDerivatives 4.0 International (CC BY-NC-ND 4.0) License. 